\title{
Low Profile Slotted Flat Waveguide Leaky Wave Antenna
}

\author{
Jan Zehentner, Jan Machac, Pavel Zabloudil \\ Czech Technical University in Prague, Technicka 2, 16627 Prague, Czech Republic
}

\begin{abstract}
This paper presents the application of a flat waveguide with a longitudinal slot in the middle of its wider wall for the design of a leaky wave antenna. The antenna radiates into one relatively narrow main beam and its gain is $14.2 \mathrm{~dB}$ at 4.75 GHz. The characteristics and radiation aspects of the antenna are discussed. The measured antenna characteristics are in accord with those predicted by simulation.
\end{abstract}

Index Terms - Flat slotted waveguide, leaky waves, leaky wave antenna, radiation.

\section{INTRODUCTION}

Low profile planar antennas have been of great interest to researchers and designers for more than thirty years. The main advantage of these antennas is that they save space and can be located on the external surface of various bodies. They are cheap, can be easily fabricated and are therefore suitable for mass production. Many kinds of planar antennas are already known and have been successfully used. The two main mechanisms of radiation are utilized. The standing wave in the resonant structure produces radiation in a narrow frequency band [1]. In contrast, the radiation of the traveling wave from the transmission line offers a wider frequency band. Traveling wave slot antennas exploiting waveguides were designed, investigated and measured fifty years ago [2]-[4]. Very sophisticated leaky wave antennas based on the stub-loaded rectangular waveguide were reported in [5], [6]. They enable independent adjustment of the beamwidth and the angle of maximum radiation. Their drawback is that they are not flat.

Open transmission lines leak power in some frequency bands and can serve as a leaky wave antenna. The microstrip leaky wave antenna was reported in [7], and the first slotline leaky wave antenna in [8]. We have successively investigated planar slotted transmission lines in order to find one leaking power only into the half-space. Our studies have included the slotline, the conductor-backed slotline and the inverted conductor-backed slotline [9]. However, all of them exhibit not only the required leakage into the space, but also undesirable leakage into the substrate. Consequently, a transmission line suitable for the design of a planar leaky wave antenna must have a substrate with finite width and thickness, shielded on the side and bottom walls and having a longitudinal slot on the top. The flat slotted waveguide (FSW) [10] meets all these demands.

An FSW leaky wave antenna fed through a CPW terminated by a narrow patch was designed and fabricated. This feeder excites the first leaky mode with odd symmetry. The antenna should therefore radiate one main beam frequency steered. However, it turned out that the bound mode was very strongly excited simultaneously with the desired leaky mode, and this substantially affected the radiation pattern. Additional modification of the feeding structure reduced this problem, and the measured antenna characteristics, including the radiation pattern, are in accord with those predicted by the EM solver.

\section{DESIGN OF THE ANTENNA}

The FSW can be viewed as a conductor-backed slotline with a finitely wide substrate metallized on its sided walls. The cross-section of the line, with proportions and co-ordinates, is shown in Fig. 1. Generally, $\varepsilon_{\mathrm{r} 2}>\varepsilon_{\mathrm{r} 1}$. When $b$ is substantially greater than $h$ we have a flat waveguide with a slot cut in the waveguide wall parallel to its longitudinal axis. This line was investigated by the method of moments applied in the spectral domain [10]. For antenna design, the complete dispersion characteristics of this line are needed. They were calculated by the method described in [10] using the APTL program [11]. In our experiment we used cheap plexiglass substrate $8 \mathrm{~mm}$ in thickness with permittivity $\varepsilon_{\mathrm{r} 2}=2.6$. To get the radiation in the frequency band between 4 and $5.5 \mathrm{GHz}$ we chose a line with dimensions $\mathrm{b}=38 \mathrm{~mm}, \mathrm{~h}=8 \mathrm{~mm}, \mathrm{w}=25 \mathrm{~mm}$.

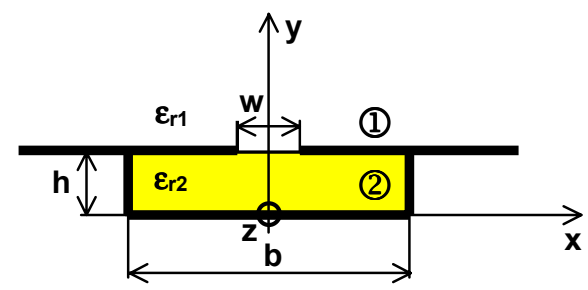

Fig. 1 Cross-section of the flat slotted waveguide.

The antenna is fed axially via a coaxial connector and the CPW terminated by a narrow patch, as shown in the antenna layout in Fig. 2. This feeder ensures excitation of the modes with odd symmetry.

The layout of the antenna feeder was optimized by the CST Microwave Studio (CST MWS), targeting the lowest $\mathrm{S}_{11}$ in the above-mentioned frequency band. The length and the width of the antenna, the feeding patch and the FSW termination determine the shape of the radiation pattern. The FSW is short-circuited at the side of the feeder and open-circuited at its end. The antenna length $l$ is $450 \mathrm{~mm}$, due to the requirement that the amplitude of the odd space leaky mode at the end of the antenna should be $5 \%$ of its magnitude on the feeding tip. The final antenna width $g$ was chosen five times wider than the FSW width $b$. 


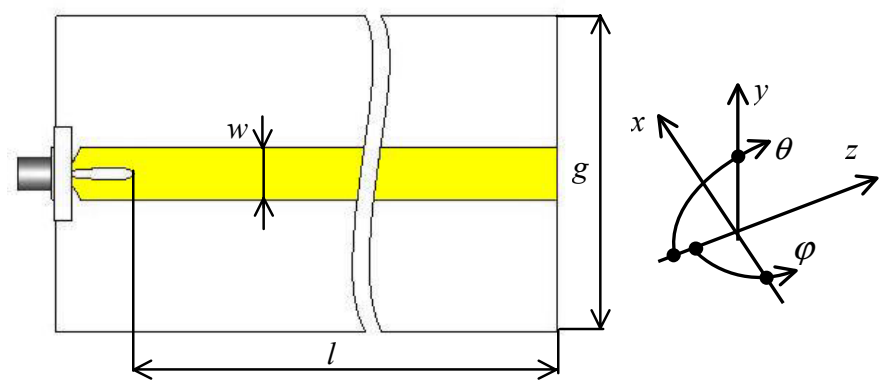

Fig. 2 Layout of the FSW leaky wave antenna.

\section{MEASUREMENTS AND DISCUSSION}

Due to the very simple antenna fabrication technology the real proportions of the CPW and the feeding patch differ from the theoretically designed values. Therefore all results presented below both computed and measured, assume the real size of the antenna and its feeder structure.

The measured frequency dependent $\mathrm{S}_{11}$ and the value calculated by CST MWS are plotted in Fig. 3. The frequency band defined by reflection losses lower than $-10 \mathrm{~dB}$ is from 4.2 to $5.2 \mathrm{GHz}$. The real and simultaneously dominant part of the antenna input impedance is greater than $80 \%$ of its total value, since $\left|S_{11}\right|<0.18$ as shown in Fig. 4. Consequently the power is radiated into space with 0.75 efficiency provided by CST MWS. Calculating the antenna circuit and radiation parameters, we set the conductivity of the aluminum $\sigma=3.5 .10^{7} \mathrm{~S} / \mathrm{m}$, and the loss factor $\operatorname{tg} \delta=0.01$ at $4.75 \mathrm{GHz}$.

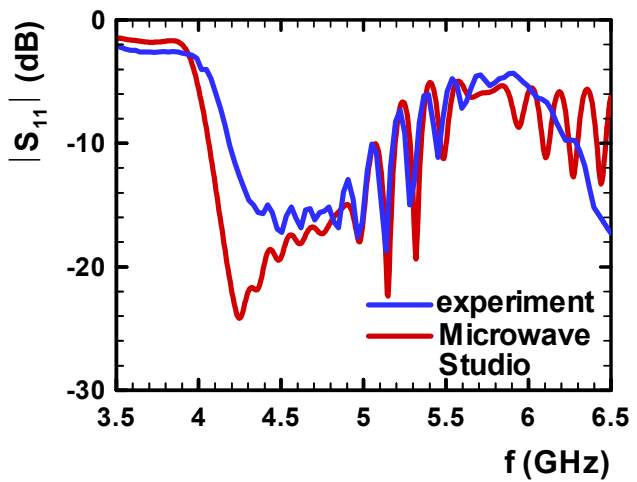

Fig. 3 Measured and calculated return losses of the FSW antenna.

The antenna radiation patterns $\left|E_{\theta}\right|$ measured in the vertical (yz)-plane and calculated by CST MWS at the frequency 4.75 $\mathrm{GHz}$ are plotted in Fig. 5. The radiation patterns are drawn in the spherical coordinates shown in Fig. 2. The main lobe tilts towards the $\mathrm{z}$ axis from $19^{\circ}$, read from the horizontal (xz)plane at $4.2 \mathrm{GHz}$, to $9^{\circ}$ at $5.2 \mathrm{GHz}$. The measured antenna gain at $4.75 \mathrm{GHz}$ is $14.2 \mathrm{~dB}$ and the calculated directivity is $15.47 \mathrm{~dB}$. The measured and calculated radiation patterns in the (xz)-plane are shown in Fig. 6.

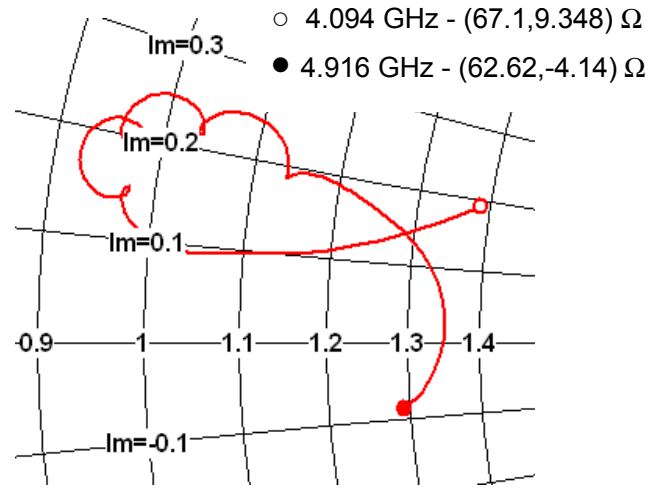

Fig. 4 The reflection coefficient $S_{11}$ of the antenna.

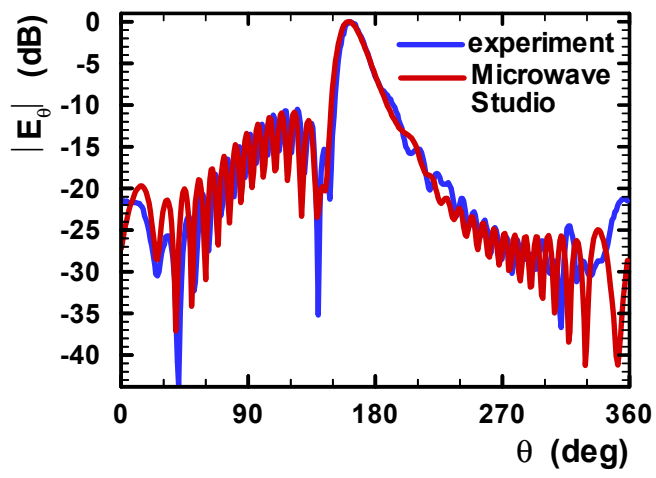

Fig. 5 Radiation patterns of the FSW antenna at $4.75 \mathrm{GHz}$ in the (yz)-plane.

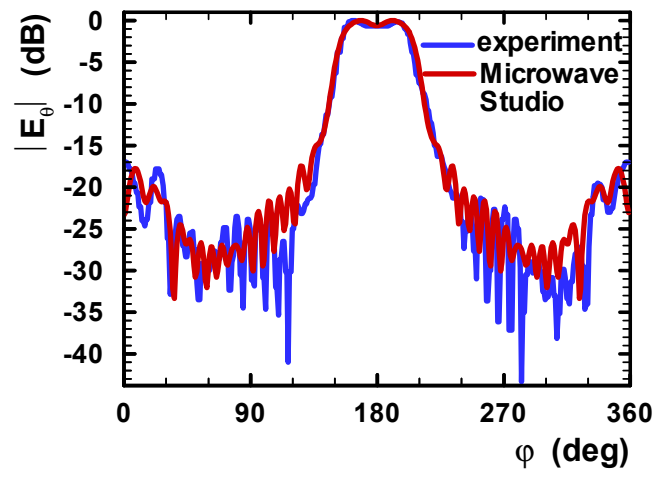

Fig. 6 Measured and calculated radiation pattern of the FSW antenna in the plane (xz) at $4.75 \mathrm{GHz}$.

Unfortunately, the unexpectedly strong excitation of the odd bound mode resulted in a standing wave along the slot, and distorted the radiation pattern. The presence of this mode is documented by the dispersion characteristics shown in Fig. 7. We determined the phase constants of the first odd space leaky mode and the odd bound mode also by measuring the wavelength of the standing wave along the line. The measured values shown as dots in Fig. 7 fit well the calculated phase constants. 
We did not anticipate such effective excitation of the odd bound mode. Its field distribution resembles the field of the odd space leaky mode, as shown in Figs. 8 and 9. The PBG structure cannot suppress the bound mode since its phase constant does not differ much from the space leaky mode phase constant. It turned out that an auxiliary absorbing shield positioned in the vicinity and around the CPW patch shown in Fig. 10 reduced the bound mode. The absorber, with conductivity $\sigma=0.3 \mathrm{~S} / \mathrm{m}$ and permittivity $\varepsilon_{\mathrm{r}}=1.25$, slightly

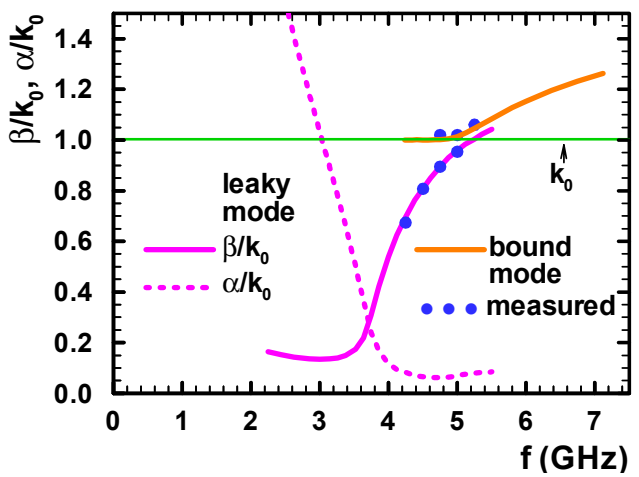

Fig. 7 Normalized dispersion characteristics of the first odd space leaky mode, and of the odd bound mode.

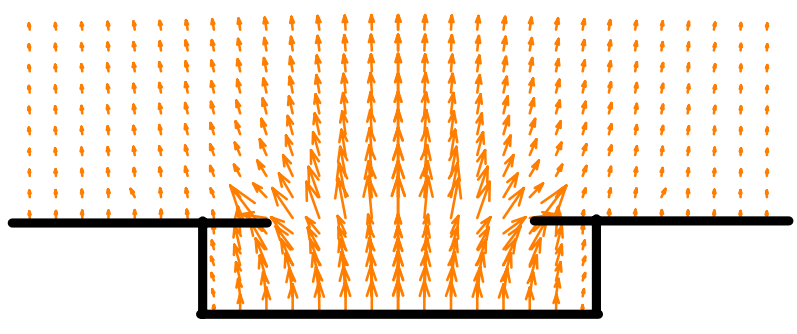

Fig. 8 Electrical field distribution of the odd bound mode on the plane perpendicular to the line axis at $5 \mathrm{GHz}$.

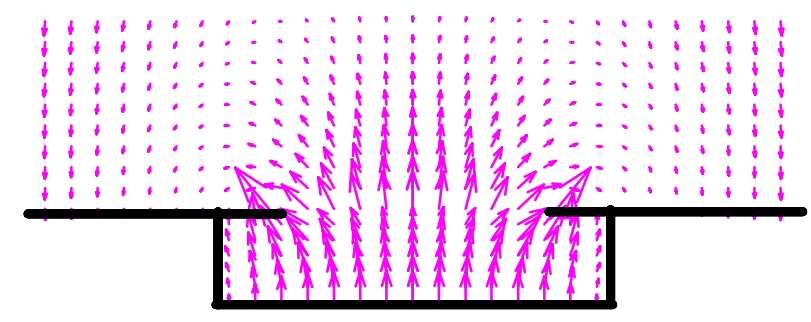

Fig. 9 Electrical field distribution of the first odd space leaky mode on the plane perpendicular to the line axis at $5 \mathrm{GHz}$.

influences the space leaky mode, since the mode evolves only in this near-zone of the patch. Now the radiation pattern has one main lobe in the (yz)-plane and the side lobes are below the main lobe by at least $-15 \mathrm{~dB}$. The measured radiation pattern agrees well with that calculated by CST MWS, as shown in Fig. 11. The suppression of the side lobes is now better by $4 \mathrm{~dB}$ compared with the original antenna structure. The calculated reflection coefficient $S_{11}$ and its magnitude $\left|S_{11}\right|$ are plotted in Figs. 12 and 13. Now the calculated efficiency 0.6 is lower than in the former case due to losses in the absorbing shield. The corresponding directivity is $14.12 \mathrm{~dB}$ while the gain is $12 \mathrm{~dB}$.

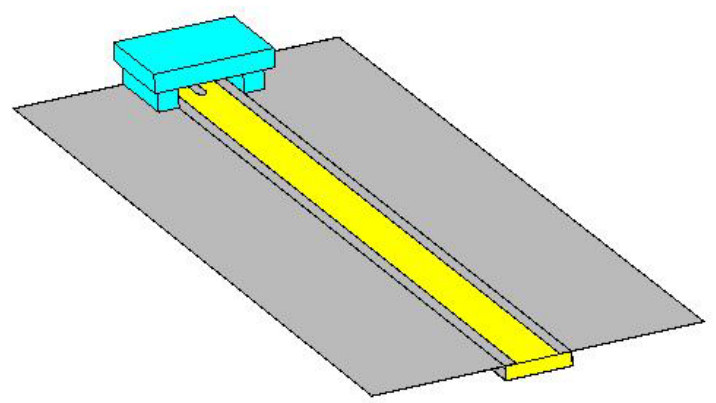

Fig. 10 Scheme of the modified FSW leaky wave antenna.

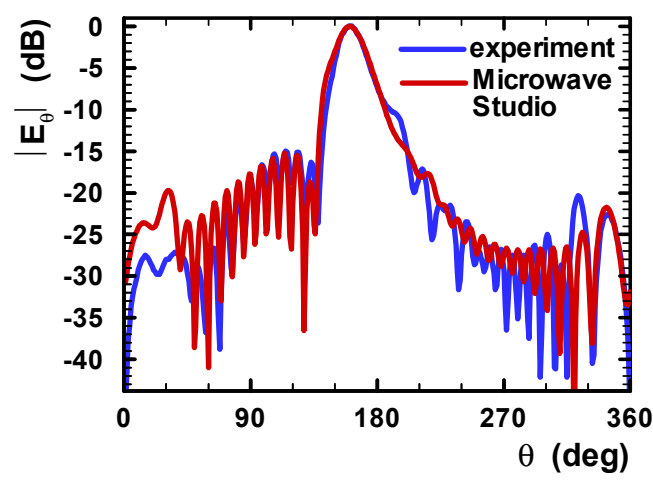

Fig. 11 Radiation pattern of the modified FSW leaky wave antenna at $4.6 \mathrm{GHz}$ in the (yz)-plane.

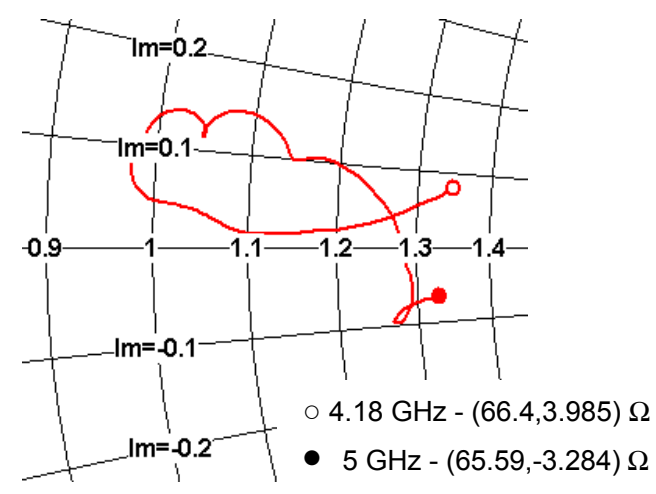

Fig. 12 The calculated reflection coefficient $S_{11}$ of the modified antenna. 
From the recorded $\mathrm{E}_{\mathrm{x}}$ field component along the line at the edge of the slot follows that the space leaky mode loses power due to the leakage and losses in the conductors and dielectric of the antenna. The bound mode is attenuated also due to the low quality of the antenna structure, but radiates less than the leaky mode. Its reflection from the antenna end creates a standing wave. This is responsible for the ripples of the radiation pattern. The estimated attenuation constant of the leaky mode from the measurement is $0.8 \mathrm{~dB} / \mathrm{cm}$, and the calculated leakage constant is $0.6 \mathrm{~dB} / \mathrm{cm}$. The attenuation constant of the bound mode can be estimated approximately $0.5 \mathrm{~dB} / \mathrm{cm}$. The finite size of the antenna resulted in radiation also below the (xz) plane. We can conclude, taking into consideration all frequency dependent antenna parameters, that its usable frequency band is approximately $10 \%$ at frequency $4.6 \mathrm{GHz}$.

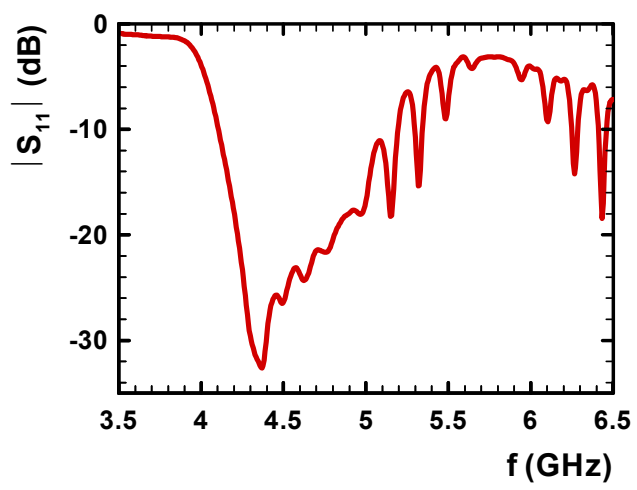

Fig. 13 Calculated return loss of the modified FSW antenna.

\section{CONCLUSIONS}

The flat slotted waveguide leaky wave antenna has been designed and fabricated. The CST MWS optimized the antenna layout. The operation frequency band defined by a reflection coefficient lower than $-10 \mathrm{~dB}$ is from 4.2 to 5.2 GHz. The antenna is fed through the CPW terminated by a narrow patch exciting the first odd leaky modes. The antenna radiates one main beam only slightly frequency steered from $19^{\circ}$ to $9^{\circ}$ when the frequency changes from $4.2 \mathrm{GHz}$ to 5.2 $\mathrm{GHz}$. The full width at half power of the main antenna beam in the (yz)-plane varies round $21^{\circ}$. The radiation pattern in the (xz)-plane is remarkably wider than the pattern in the (yz)plane. The finite proportions of the ground conductors are responsible for radiation below the antenna plane. The antenna radiation pattern is affected by the odd bound mode simultaneously excited with the odd space leaky mode. The standing wave produced by the bound mode causes a ripple in the radiation pattern.
An additional absorbing shield above the CPW feeding patch reduced the ripple and improved the offset of the side lobes by $-4 \mathrm{~dB}$ to $-15 \mathrm{~dB}$ at $4.6 \mathrm{GHz}$. The gain is $12 \mathrm{~dB}$ at 4.6 $\mathrm{GHz}$. Another feeder, probably a slot in the waveguide wall, would ensure a fully planar version of the antenna. Improved technology using gold metallization and a more precise layout will surely lower the own antenna losses and enhance its gain and efficiency. The antenna demonstrated the ability of FSW to leak into space. The FSW leaky wave antenna may be useful for applications where small angles of elevation above a large conducting surface are required.

\section{ACKNOWLEDGEMENT}

This work has been supported by the Grant Agency of the Czech Republic under project 102/03/0449 "New circuit devices for communication technology".

\section{REFERENCES}

[1] I. J. Bahl and P. Bhartia, Microstrip Antennas, Dedham: Artech House, 1980.

[2] J. N. Hines, V. H. Rumsey and C. H. Walter, "Traveling-Wave Slot Antennas," Proc. IRE, vol. 41, no. 11, pp. 1624-1631, November 1953.

[3] L. O. Goldstone and A. A. Oliner, "Leaky-Wave Antennas I: Rectangular Waveguides," IRE Trans. Antennas \& Propagation, vol. 7, no. 10, pp. 307-319, October 1959.

[4] F. J. Zurker: "Surface and leaky-wave antennas," in Antenna Engineering Handbook, H. Jasik, ed., New York: McGrew Hill, 1961.

[5] P. Lampariello, F. Frezza, H. Shigesava, M. Tsuji and A. A. Oliner, "A Versatile Leaky-Wave Antenna Based on Stubloaded Rectangular Waveguide: Part I - Theory," IEEE Transactions on Antennas and Propagation, vol. 46, no. 7, pp. 1032-1041, July 1998.

[6] F. Frezza, P. Lampariello, H. Shigesava, M. Tsuji and A. A. Oliner, "A Versatile Leaky-Wave Antenna Based on Stubloaded Rectangular Waveguide: Part II - Effects of Flanges and Finite Stub Length," IEEE Transactions on Antennas and Propagation, vol. 46, no. 7, pp. 1042-1046, July 1998.

[7] W. Menzel: "A New Traveling-Wave Antenna in Microstrip," Arch. Electr. Uebertrag. Tech., Vol. 33, pp. 137-140, April 1979.

[8] J.-W. Sheen and Y.-D. Lin, " Propagation Characteristics of the Slotline First Higher Order Mode," IEEE Transactions on Microwave Theory and Techn., vol. 46, no. 11, pp. 1774-1781, November 1998.

[9] J. Macháč, J. Zehentner and J. Hruška, "Conductor-Backed Slotline Antenna," Proceedings of the 34th European Microwave Conference, vol. 2, pp. 1205-1208, October 2004.

[10] J. Zehentner, J. Macháč and J. Mrkvica, "Flat Waveguide with a Longitudinal Slot," 2005 IEEE MTT-S International Microwave Symposium Digest, TH4C-4, June 2005.

[11] J. Mrkvica, "Wave propagation on modified slotlines and coplanar lines," $\mathrm{PhD}$ Thesis (in Czech), Prague: CTU in Prague, 2004. 